PROCEEDINGS OF THE

AMERICAN MATHEMATICAL SOCIETY

Volume 131, Number 12, Pages 3873-3881

S 0002-9939(03)06977-6

Article electronically published on April 24, 2003

\title{
POSITIVE HARMONIC FUNCTIONS OF FINITE ORDER IN A DENJOY TYPE DOMAIN
}

\author{
HIROAKI AIKAWA \\ (Communicated by Juha M. Heinonen) \\ Dedicated to Professor Kaoru Hatano on the occasion of his 60th birthday
}

\begin{abstract}
We introduce a Denjoy type domain and prove that the dimension of the cone of positive harmonic functions of finite order in the domain with vanishing boundary values is one or two, whenever the boundary is included in a certain set.
\end{abstract}

\section{INTRODUCTION}

Let $E$ be a closed set in the hyperplane $\left\{x=\left(x_{1}, \ldots, x_{n}\right): x_{n}=0\right\}$ of the Euclidean space $\mathbb{R}^{n}$ with $n \geq 2$. The complement of $E$ is called a Denjoy domain. Benedicks [5] studied positive harmonic functions in a Denjoy domain vanishing on the boundary. He proved that the dimension of such positive harmonic functions is one or two and gave a distinction theorem in terms of the harmonic measure of $E$. A number of studies have been made after Benedicks' work. Many of them treated the case when $E$ lies in more general sets, such as smooth and Lipschitz surfaces, finitely many rays leaving the origin. There are many estimates of the dimension of positive harmonic functions in such domains vanishing on the boundaries. See e.g. [3, 6], 7], 9], 11], [13, 14.

Poggi-Corradini [12] considered a somewhat different extension. He relaxed the constraint that $E$ lies in the hyperplane to that $E$ lies in a strip $\{-1 / 2<\operatorname{Im} z<0\}$. In this circumstance, the family of all positive harmonic functions vanishing on $E$ is too complicated to be described; its dimension may be infinite. However, if one restricts his attention to these functions of finite order, then one may get some results. To be precise, we use the following notation. By $B(x, r)$ and $S(x, r)$ we denote the open ball and the sphere with center at $x$ and radius $r$, respectively. If $x=0$, then we write $B(r)$ and $S(r)$ for simplicity. Let $u$ be a positive harmonic function on an unbounded domain $\Omega$. We say that $u$ is of order $\lambda$ if

$$
\lambda=\limsup _{r \rightarrow \infty} \frac{\log \left(\sup _{\Omega \cap B(r)} u\right)}{\log r} .
$$

Received by the editors May 31, 2002 and, in revised form, August 6, 2002.

2000 Mathematics Subject Classification. Primary 31A05, 31B05, 31B25.

Key words and phrases. Denjoy type domain, harmonic function, finite order.

This work was supported in part by Grant-in-Aid for Scientific Research (A) (No. 11304008), (B) (No. 12440040) and Exploratory Research (No. 13874023) Japan Society for the Promotion of Science. 
If $\lambda<\infty$, then $u$ is said to be of finite order. See Hayman-Kennedy 10, Definition 4.1]. By $\omega(x, E, U)$ we denote the harmonic measure of $E$ for an open set $U$ evaluated at $x$. By the symbol $A$ we denote a positive constant whose value is unimportant and may change even in the same line. If necessary, we use $A_{1}, A_{2}, \ldots$, to specify them. Poggi-Corradini proved the following.

Theorem A. Suppose $\Omega=\mathbb{C} \backslash E$ is a planar domain with $E \subset\{-1 / 2<\operatorname{Im} z<0\}$ of positive capacity and such that $\partial \Omega$ is regular. Let $\mathcal{P}$ be the family of positive harmonic functions on $\Omega$ vanishing on $\partial \Omega$ and $\mathcal{F}$ the subfamily of functions of finite order.

(i) Suppose $u \in \mathcal{P}$. Then $u \in \mathcal{F}$ if and only if

$$
\limsup _{r \rightarrow \infty} \omega(i, S(r), \Omega \cap B(r)) \max _{S(r)} u<\infty .
$$

Moreover, in this case $\max _{S(r)} u \leq A r$.

(ii) There are either one or two linearly independent, minimal positive harmonic functions, vanishing on $\partial \Omega$, equal to 1 at $i$, and of finite order (briefly put $\operatorname{dim} \mathcal{F}=1$ or 2 ).

(iii) If

$$
\sup _{x \in \mathbb{R}} \max \{\omega(x, \mathbb{R}+i, \Omega), \omega(x, \mathbb{R}-i, \Omega)\}<1,
$$

then $\operatorname{dim} \mathcal{F}=2$.

(iv) Suppose that $\Omega+1=\Omega$. Then $\mathcal{P}=\mathcal{F}$.

We remark that the regularity of $\partial \Omega$ can be removed; the boundary condition of $u$ can be generalized as follows: $u=0$ on $\partial \Omega$ outside a polar set and $u$ is locally bounded near $\partial \Omega$. In this paper we show that some parts of Theorem $\AA$ hold for a certain domain $\Omega \subset \mathbb{R}^{n}$ whose complement is included in a set wider than a strip. We shall say that such a domain is of Denjoy type.

Theorem 1. Let $n \geq 2$. Let $\Omega=\mathbb{R}^{n} \backslash E$ be a domain such that

$$
E \subset\left\{x \in \mathbb{R}^{n}:\left|x_{n}\right| \leq f\left(\left|x^{\prime}\right|\right) \text { with } x^{\prime}=\left(x_{1}, \ldots, x_{n-1}\right)\right\},
$$

where $f(t)$ is a nonnegative function for $t \geq 0$ with

$$
\lim _{t \rightarrow \infty} \frac{f(t)}{t}=0 .
$$

Let $\mathcal{P}$ be the family of positive harmonic functions on $\Omega$ vanishing on $\partial \Omega$ outside a polar set and locally bounded near $\partial \Omega$. Let $\mathcal{F}$ be the subfamily of functions of finite order.

(i) Suppose $u \in \mathcal{P}$. Then $u \in \mathcal{F}$ if and only if $u$ is of order at most 1 .

(ii) $\operatorname{dim} \mathcal{F}=1$ or 2 .

\section{Positive harmonic Functions on a CONE}

Poggi-Corradini [12] used a lemma modeled after Ancona [2, Lemme 1], whose argument is based on the reflection of the domain. In our situation, the complement is not included in a strip, so that the reflection argument cannot be applied. Instead, we shall invoke some estimates of harmonic measures on cones. Write the Laplacian as

$$
\Delta=\frac{n-1}{r} \frac{\partial}{\partial r}+\frac{\partial^{2}}{\partial r^{2}}+\frac{1}{r^{2}} \Lambda_{n}
$$




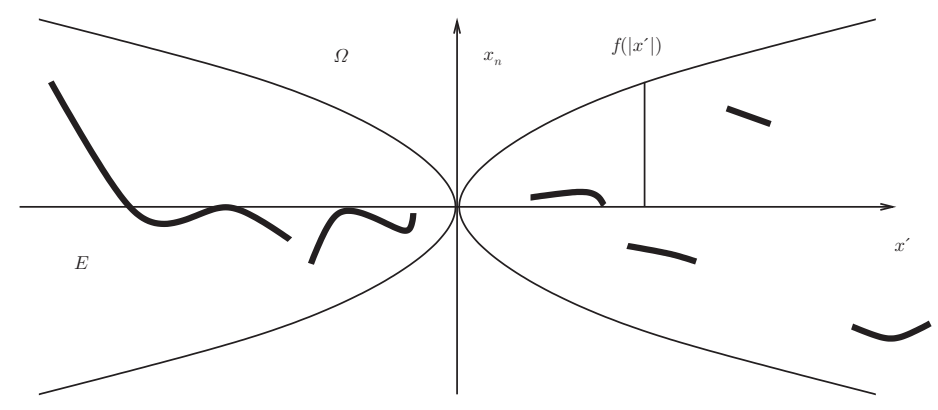

Figure 1. Denjoy type domain

where $\Lambda_{n}$ is the Laplace-Beltrami operator. Let $U$ be a (relatively) open set on the unit sphere $S(1)$. Consider the Laplace-Beltrami equation

$$
\begin{aligned}
\Lambda_{n} F+\lambda F=0 & \text { on } U, \\
F=0 & \text { on } \partial U,
\end{aligned}
$$

where $\lambda=\lambda(U)$ is the first positive eigenvalue. We let $F_{U}$ be the positive eigenfunction corresponding to $\lambda$. The characteristic constant $\alpha=\alpha(U)$ is defined by the positive root of the equation

$$
\alpha(\alpha+n-2)=\lambda .
$$

Let $\Gamma(U)=\left\{x \in \mathbb{R}^{n}: x /|x| \in U\right\}$ be the cone subtended by $U$ with vertex at the origin. Then it is easy to see that

$$
h_{U}(x)=|x|^{\alpha} F_{U}\left(\frac{x}{|x|}\right)
$$

is a positive harmonic function on $\Gamma(U)$ vanishing on $\partial \Gamma(U)$, provided $U$ is sufficiently smooth. In fact, $\Gamma(U)$ has one Martin boundary at infinity and $h_{U}$ corresponds to the Martin kernel at infinity. If $U$ is the spherical cap $\Sigma(\theta)=\{x \in S(1)$ : $\left.x_{n}>\cos \theta\right\}$, then we write $\Gamma(\theta)$ and $\alpha(\theta)$ for $\Gamma(\Sigma(\theta))$ and $\alpha(\Sigma(\theta))$. It is known that a spherical cap has the least characteristic constant among open sets on $S(1)$ with the same surface measure (Sperner [15]). Friedland and Hayman [8] gave a very precise estimate of the characteristic constants. For spherical caps we can easily observe that $\alpha(\theta)$ is a strictly decreasing function of $\theta$ such that $\alpha(\pi / 2)=1$ and $\alpha(\theta) \uparrow \infty$ as $\theta \downarrow 0$. From these observations we have the following estimates of the harmonic measure of a spherical cone.

Lemma 1. Let $0<\theta_{1}<\theta_{2}<\theta_{3}<\pi / 2$.

(i) There is a constant $0<A_{1}<1$ depending only on $\theta_{1}$ and $\theta_{2}$ such that

$$
\omega\left(\cdot, \Gamma\left(\theta_{2}\right) \cap S(r), \Gamma\left(\theta_{2}\right) \cap B(r)\right) \geq A_{1} \quad \text { on } \overline{\Gamma\left(\theta_{1}\right) \cap B(r)} \backslash B(r / 2)
$$

for $r>0$.

(ii) There is a constant $A_{2}>1$ depending only on $\theta_{1}$ and $\theta_{2}$ such that

$$
A_{2}^{-1}\left(\frac{|x|}{r}\right)^{\alpha} \leq \omega\left(x, \Gamma\left(\theta_{1}\right) \cap S(r), \Gamma\left(\theta_{3}\right) \cap B(r)\right) \leq A_{2}\left(\frac{|x|}{r}\right)^{\alpha}
$$

for $r>0$ and $x \in \overline{\Gamma\left(\theta_{2}\right) \cap B(r / 2)}$, where $\alpha=\alpha\left(\theta_{3}\right)>1$ is the characteristic constant of $\Sigma\left(\theta_{3}\right)$ such that $\alpha\left(\theta_{3}\right) \downarrow 1$ as $\theta_{3} \uparrow \pi / 2$. 
We also need an estimate of the harmonic measure of a non-spherical cone.

Lemma 2. Let $U(\varphi)=\left\{x \in S(1):\left|x_{n}\right|<\sin \varphi\right\}$ and let $V(\varphi)=\left\{x:\left|x_{n}\right|<\right.$ $|x| \sin \varphi\}$ be the cone subtended by $U(\varphi)$ for $0<\varphi<\pi / 2$. Let $\beta$ be a positive constant less than the characteristic constant $\alpha(U(\varphi))$ of $U(\varphi)$. Then there is a positive constant $A_{3}$ depending only on $\varphi$ and $\beta$ such that

$\omega(x, \partial V(\varphi) \cap B(2 r) \backslash B(r), V(\varphi)) \leq \omega(x, V(\varphi) \cap S(r), V(\varphi) \cap B(r)) \leq A_{3}\left(\frac{|x|}{r}\right)^{\beta}$

for $x \in V(\varphi) \cap B(r)$.

Proof. The first inequality is obvious by the maximum principle. Let $\varphi^{\prime}>\varphi$ be so close to $\varphi$ that the characteristic constant $\alpha^{\prime}$ of $U\left(\varphi^{\prime}\right)$ satisfies $\beta \leq \alpha^{\prime}<\alpha(U(\varphi))$. Consider the harmonic function $h_{U\left(\varphi^{\prime}\right)}$ given by (11). Then we have

$$
A^{-1}|x|^{\alpha^{\prime}} \leq h_{U\left(\varphi^{\prime}\right)}(x) \leq A|x|^{\alpha^{\prime}} \text { for } x \in V(\varphi),
$$

where $A$ is independent of $x$. The maximum principle and the left inequality of (2) yield

$$
\omega(\cdot, V(\varphi) \cap S(r), V(\varphi) \cap B(r)) \leq A r^{-\alpha^{\prime}} h_{U\left(\varphi^{\prime}\right)} \quad \text { on } V(\varphi) \cap B(r),
$$

so that

$$
\omega(x, V(\varphi) \cap S(r), V(\varphi) \cap B(r)) \leq A\left(\frac{|x|}{r}\right)^{\alpha^{\prime}} \leq A\left(\frac{|x|}{r}\right)^{\beta} \quad \text { for } x \in V(\varphi) \cap B(r)
$$

by the right inequality of (2).

The following estimate of the harmonic measure of a ball is easily proved by dilation.

Lemma 3. For each $\eta>0$ there is $\varepsilon>0$ such that if $0<\varphi<\varepsilon$, then

$$
\omega(\cdot, V(\varphi) \cap S(r), B(r))<\eta \quad \text { on } B(0, r / 2)
$$

for $r>0$.

By the repeated application of the Harnack inequality along a Harnack chain, we obtain the following lemma. For a proof see [1, Lemma 11], where more general John domains are treated.

Lemma 4. For $0<\theta<\pi / 2$ there are positive constants $\gamma(\theta)$ and $A_{4}$ depending only on $\theta$ such that if $1 \leq r \leq R / 2$ and $h$ is a positive harmonic function on a truncated cone $\Gamma(\theta) \cap B(R)$, then

$$
\frac{h(0, \ldots, 0, r)}{h(0, \ldots, 0,1)} \leq A_{4} r^{\gamma} .
$$

Moreover, $\gamma(\theta) \downarrow 1$ as $\theta \uparrow \pi / 2$.

\section{Proof of Theorem 1}

Let us begin with an estimate of a harmonic measure for $\Omega$. Without loss of generality, we may assume that $f$ is nondecreasing, $f(t)=0$ for $0 \leq t \leq 1$ and $B(2) \subset \Omega$. 
Lemma 5. Let $0<\theta \leq \pi$. Then

$$
\liminf _{r \rightarrow \infty} r^{1+\eta} \omega(0, \Gamma(\theta) \cap S(r), \Omega \cap B(r))=\infty
$$

for each $\eta>0$.

Proof. In view of Lemma 1 we take $0<\theta_{3}<\pi / 2$ so close to $\pi / 2$ that $\alpha=\alpha\left(\theta_{3}\right)<$ $1+\eta$. Taking $\theta$ smaller makes the harmonic measure smaller, so we may assume that $0<\theta<\theta_{3}$ and $\Gamma(\theta) \subset \Omega$. By assumption there is $t_{0}>0$ such that

$$
\frac{f(t)}{t} \leq \cot \theta_{3} \quad \text { for } t \geq t_{0} .
$$

Let $z=\left(0, \ldots, 0, f\left(t_{0}\right)\right)$. Then we have

$$
z+\Gamma\left(\theta_{3}\right) \subset\left\{x: x_{n}>f\left(\left|x^{\prime}\right|\right)\right\} \subset \Omega .
$$

Let $0<\theta_{1}<\theta$ and $r \geq 4 f\left(t_{0}\right)$. Then

$$
\left(z+\Gamma\left(\theta_{1}\right)\right) \cap S\left(z, r-f\left(t_{0}\right)\right) \subset \Gamma\left(\theta_{1}\right) \cap \overline{B(r)} \backslash B(r / 2),
$$

and on the set of the right-hand side

$$
\omega(\cdot, \Gamma(\theta) \cap S(r), \Omega \cap B(r)) \geq \omega(\cdot, \Gamma(\theta) \cap S(r), \Gamma(\theta) \cap B(r)) \geq A_{1}
$$

by Lemma 1 (i), where $A_{1}$ depends only on $\theta$ and $\theta_{1}$. Hence (3) and the maximum principle yield

$$
\begin{aligned}
& \omega(\cdot, \Gamma(\theta) \cap S(r), \Omega \cap B(r)) \\
& \geq A_{1} \omega\left(\cdot,\left(z+\Gamma\left(\theta_{1}\right)\right) \cap S\left(z, r-f\left(t_{0}\right)\right),\left(z+\Gamma\left(\theta_{3}\right)\right) \cap B\left(z, r-f\left(t_{0}\right)\right)\right)
\end{aligned}
$$

on $\left(z+\Gamma\left(\theta_{3}\right)\right) \cap B\left(z, r-f\left(t_{0}\right)\right)$. Letting $z^{*}=\left(0, \ldots, 0,2 f\left(t_{0}\right)\right)$, we obtain from Lemma 1 (ii) and translation that

$$
\omega\left(z^{*}, \Gamma(\theta) \cap S(r), \Omega \cap B(r)\right) \geq \frac{A_{1}}{A_{2}}\left(\frac{f\left(t_{0}\right)}{r-f\left(t_{0}\right)}\right)^{\alpha} .
$$

By the Harnack inequality

$$
\omega(0, \Gamma(\theta) \cap S(r), \Omega \cap B(r)) \geq A \omega\left(z^{*}, \Gamma(\theta) \cap S(r), \Omega \cap B(r)\right) \quad \text { for } r \geq 4 f\left(t_{0}\right),
$$

where $A$ may depend on $f\left(t_{0}\right)$ but not on $r$. Hence

$$
r^{1+\eta} \omega(0, \Gamma(\theta) \cap S(r), \Omega \cap B(r)) \geq A r^{1+\eta}\left(\frac{f\left(t_{0}\right)}{r-f\left(t_{0}\right)}\right)^{\alpha} \rightarrow \infty
$$

as $r \rightarrow \infty$. Thus the lemma follows.

We use the following notation in the proof of Theorem 1 Let $I^{+}(\varphi)=\{x$ : $\left.x_{n}>|x| \sin \varphi\right\}, I^{-}(\varphi)=\left\{x: x_{n}<-|x| \sin \varphi\right\}$ and $I(\varphi)=I^{+}(\varphi) \cup I^{-}(\varphi)$ for $0<\varphi<\pi / 2$. Then $I^{+}(\varphi)=\Gamma\left(\frac{\pi}{2}-\varphi\right)$ and $I(\varphi)=\mathbb{R}^{n} \backslash \overline{V(\varphi)}$ in the notation in the previous section.

Proof of Theorem 1 (i). It is sufficient to show that if $u \in \mathcal{F}$, then $u$ is of order at most 1. Let $u \in \mathcal{F}$ and let $M_{j}=\sup _{\Omega \cap B\left(2^{j}\right)} u$. Then there is $S>1$ such that

$$
M_{j} \leq S^{j} \text { for } j \geq 0 .
$$

Let $\eta>0$. We shall show that

$$
M_{j} \leq A 2^{(1+\eta) j} \quad \text { for sufficiently large } j,
$$

where $A>0$ may depend on $u$ and $\eta$, but not on $j$. Then the arbitrariness of $\eta>0$ implies that $u$ is of order at most 1 . 
In view of Lemma 3 we find $\varphi>0$ such that for every $j \geq 0$,

$$
\omega\left(\cdot, S\left(2^{j+1}\right) \backslash I(\varphi), B\left(2^{j+1}\right)\right) \leq \frac{1}{2^{1+\eta} S} \quad \text { on } B\left(2^{j}\right) .
$$

By assumption we may assume that $S(r) \cap I\left(\varphi^{\prime}\right) \subset \Omega$ for large $r$, say $r \geq 2^{j_{0}}$, with $0<\varphi^{\prime}<\varphi$. Hereafter let $j>j_{0}$. By the maximum principle over $\Omega \cap B\left(2^{j+1}\right)$ we have

$$
\begin{aligned}
u & \leq M_{j+1} \omega\left(\cdot, \Omega \cap S\left(2^{j+1}\right) \backslash I(\varphi), \Omega \cap B\left(2^{j+1}\right)\right)+\sup _{S\left(2^{j+1}\right) \cap I(\varphi)} u \\
& \leq \frac{1}{2^{1+\eta} S} M_{j+1}+\sup _{S\left(2^{j+1}\right) \cap I(\varphi)} u \quad \text { on } \Omega \cap B\left(2^{j}\right) .
\end{aligned}
$$

The Harnack inequality and Lemma 5 yield

$$
u(0) \geq \omega\left(0, I^{+}(\varphi) \cap S\left(2^{j}\right), \Omega \cap B\left(2^{j}\right)\right) \inf _{I^{+}(\varphi) \cap S\left(2^{j}\right)} u \geq A 2^{-(1+\eta) j} \sup _{I^{+}(\varphi) \cap S\left(2^{j+1}\right)} u
$$

for sufficiently large $j$, say $j \geq j_{1}$. Similarly, $\sup _{I^{-}(\varphi) \cap S\left(2^{j+1}\right)} u$ is estimated, and hence

$$
u(0) \geq A 2^{-(1+\eta) j} \sup _{I(\varphi) \cap S\left(2^{j+1}\right)} u .
$$

Substituting this estimate to (6) and taking the supremum over $\Omega \cap B\left(2^{j}\right)$, we obtain

$$
M_{j} \leq \frac{1}{2^{1+\eta} S} M_{j+1}+A 2^{(1+\eta) j} u(0),
$$

so that

$$
\begin{aligned}
2^{-(1+\eta) j} M_{j} & \leq \frac{1}{S} 2^{-(1+\eta)(j+1)} M_{j+1}+A u(0) \\
& \leq \frac{1}{S}\left(\frac{1}{S} 2^{-(1+\eta)(j+2)} M_{j+2}+A u(0)\right)+A u(0) \\
& =\frac{1}{S^{2}} 2^{-(1+\eta)(j+2)} M_{j+2}+A\left(1+\frac{1}{S}\right) u(0) .
\end{aligned}
$$

Repeating this, we obtain

$$
2^{-(1+\eta) j} M_{j} \leq \frac{1}{S^{k}} 2^{-(1+\eta)(j+k)} M_{j+k}+A u(0) \sum_{i=0}^{k-1} \frac{1}{S^{i}} \quad \text { for } k \geq 1 .
$$

Let $k \rightarrow \infty$. Then (4) yields

$$
2^{-(1+\eta) j} M_{j} \leq A u(0) \sum_{i=0}^{\infty} \frac{1}{S^{i}}=\frac{A}{1-1 / S} u(0) \quad \text { for } j \geq j_{1}
$$

This implies (5).

Proof of Theorem 1 (ii). By repeating the proof of Benedicks [5, Theorem 2] verbatim, we obtain $\operatorname{dim} \mathcal{F} \leq 2$ from Friedland and Hayman [8, Theorem 2]. So, let us prove $\operatorname{dim} \mathcal{F} \geq 1$.

Compare the characteristic constant $\alpha(U(\varphi))$ in Lemma 2 and the constant $\gamma$ in Lemma 4. Since $\alpha(U(\varphi)) \uparrow \infty$ and $\gamma\left(\frac{\pi}{2}-\varphi\right) \downarrow 1$ as $\varphi \downarrow 0$, we can choose $\varphi_{0}$ so small that

$$
1<\gamma_{0}=\gamma\left(\frac{\pi}{2}-\varphi_{0}\right)<\alpha\left(U\left(\varphi_{0}\right)\right)
$$


We first treat the case when $I\left(\varphi_{1}\right) \subset \Omega$ with some $\varphi_{1}, 0<\varphi_{1}<\varphi_{0}$, such that

$$
\alpha\left(\Sigma\left(\frac{\pi}{2}-\varphi_{1}\right)\right) \leq \gamma_{0},
$$

where we recall that $\alpha\left(\Sigma\left(\frac{\pi}{2}-\varphi_{1}\right)\right)$ is the characteristic constant of the spherical cap $\Sigma\left(\frac{\pi}{2}-\varphi_{1}\right)=\left\{x \in S(1): x_{n}>\cos \left(\frac{\pi}{2}-\varphi_{1}\right)\right\}$. Let $R>0$ and consider $u_{R}=\omega(\cdot, \Omega \cap S(R), \Omega \cap B(R))$. We claim that if $1<r<R$, then

$$
u_{R} \leq A r^{\gamma_{0}} u_{R}(0) \quad \text { on } \Omega \cap B(r),
$$

where $A$ is independent of $r$ and $R$.

For a moment let $1<r<R / 2$. Let $z_{r}^{+}=(0, \ldots, 0, r)$. Then $u_{R} \leq A u_{R}\left(z_{r}^{+}\right)$on $I^{+}\left(\varphi_{0}\right) \cap S(r)$ by the Harnack inequality. Moreover, Lemma 4 implies

$$
u_{R}\left(z_{r}^{+}\right) \leq A_{4} r^{\gamma_{0}} u_{R}\left(z_{1}^{+}\right) \leq A r^{\gamma_{0}} u_{R}(0),
$$

where the last inequality follows from $B(2) \subset \Omega$ and the Harnack inequality. Similarly, we estimate $u_{R}$ on $I^{-}\left(\varphi_{0}\right) \cap S(r)$ and obtain from continuity that

$$
u_{R} \leq A r^{\gamma_{0}} u_{R}(0) \quad \text { on } \overline{I\left(\varphi_{0}\right) \cap B(r)} .
$$

Actually, (9) holds for $1<r<R$ since, by Lemma 5 .

$$
R^{\gamma_{0}} u_{R}(0) \geq A,
$$

where $A$ is independent of $R$. Thus (8) holds on $\overline{I\left(\varphi_{0}\right) \cap B(r)}$.

Let us estimate $u_{R}$ on $\Omega \cap V\left(\varphi_{0}\right) \cap B(r)$. Let $v_{R}$ be the Dirichlet solution on $V\left(\varphi_{0}\right) \cap B(R)$ such that

$$
v_{R}= \begin{cases}u_{R} & \text { on } \partial V\left(\varphi_{0}\right) \cap B(R), \\ 0 & \text { on } V\left(\varphi_{0}\right) \cap S(R) .\end{cases}
$$

Then the maximum principle yields

$$
u_{R} \leq v_{R}+\omega\left(\cdot, V\left(\varphi_{0}\right) \cap S(R), V\left(\varphi_{0}\right) \cap B(R)\right) \quad \text { on } \Omega \cap V\left(\varphi_{0}\right) \cap B(R) .
$$

We have from Lemma 2, (7) and (10) that

$$
\begin{aligned}
& \omega\left(x, V\left(\varphi_{0}\right) \cap S(R), V\left(\varphi_{0}\right) \cap B(R)\right) \\
& \leq A_{3}\left(\frac{|x|}{R}\right)^{\gamma_{0}} \leq A|x|^{\gamma_{0}} u_{R}(0) \quad \text { for } x \in V\left(\varphi_{0}\right) \cap B(R) .
\end{aligned}
$$

Now what remains is to estimate $v_{R}$. It follows from (9) that

$$
u_{R}(y) \leq A|y|^{\gamma_{0}} u_{R}(0) \quad \text { for } y \in \partial V\left(\varphi_{0}\right) \cap B(R) .
$$

Therefore, if $x \in V\left(\varphi_{0}\right) \cap B(r)$, then

$$
\begin{aligned}
v_{R}(x) & \leq A r^{\gamma_{0}} u_{R}(0) \omega\left(x, \partial V\left(\varphi_{0}\right) \cap B(2 r), V\left(\varphi_{0}\right)\right) \\
& +\sum_{j=1}^{k} A\left(2^{j} r\right)^{\gamma_{0}} u_{R}(0) \omega\left(x, \partial V\left(\varphi_{0}\right) \cap B\left(2^{j+1} r\right) \backslash B\left(2^{j} r\right), V\left(\varphi_{0}\right)\right) \\
& +A\left(2^{k+1} r\right)^{\gamma_{0}} u_{R}(0) \omega\left(x, \partial V\left(\varphi_{0}\right) \cap B(R) \backslash B\left(2^{k+1} r\right), V\left(\varphi_{0}\right)\right),
\end{aligned}
$$


where $2^{k+1} r \leq R<2^{k+2} r$. We find a constant $\beta$ such that $\gamma_{0}<\beta<\alpha\left(U\left(\varphi_{0}\right)\right)$ by (7). We have from Lemma 2 that

$$
v_{R}(x) \leq A r^{\gamma_{0}} u_{R}(0)+\sum_{j=1}^{\infty} A\left(2^{j} r\right)^{\gamma_{0}} u_{R}(0)\left(\frac{r}{2^{j} r}\right)^{\beta} \leq A r^{\gamma_{0}} u_{R}(0) .
$$

Thus the claim (8) follows from (9) and (11).

Consider the family of positive harmonic functions $\left\{u_{R} / u_{R}(0)\right\}$ whose values at 0 are equal to 1 . We can choose a sequence $R_{j} \rightarrow \infty$ such that $u_{R_{j}} / u_{R_{j}}(0)$ converges to a positive harmonic function $h$. In view of (8) and the maximum principle we observe that

$$
\frac{u_{R_{j}}}{u_{R_{j}}(0)} \leq A r^{\gamma_{0}} \omega(\cdot, \Omega \cap S(r), \Omega \cap B(r)) \quad \text { on } \Omega \cap B(r),
$$

whenever $1<r<R_{j}$. Letting $j \rightarrow \infty$, we obtain

$$
h \leq A r^{\gamma_{0}} \omega(\cdot, \Omega \cap S(r), \Omega \cap B(r)) \quad \text { on } \Omega \cap B(r) .
$$

Since $r>1$ is arbitrary, it follows that $h$ is of order at most $\gamma_{0}$ and vanishes q.e. on the boundary of $\Omega$, so that $h \in \mathcal{F}$. Thus $\operatorname{dim} \mathcal{F} \geq 1$.

Finally, we consider the general case. Suppose $I\left(\varphi_{1}\right) \not \subset \Omega$. Let $\Omega^{\prime}=\mathbb{R}^{n} \backslash$ $\left(\overline{V\left(\varphi_{1}\right)} \cap \Omega^{c}\right)$. Then $\Omega^{\prime}$ is a domain including $I\left(\varphi_{1}\right)$. As above, we find a positive harmonic function $h$ of finite order in $\Omega^{\prime}$ vanishing q.e. on the boundary of $\Omega^{\prime}$. The geometry of $\Omega$ implies that $I\left(\varphi_{1}\right) \backslash \Omega$ is a bounded set. Hence the regularized reduced function $\widehat{R}_{h}^{I\left(\varphi_{1}\right) \backslash \Omega}$ with respect to $\Omega^{\prime}$ is a potential ([4, Theorem 5.3.5]) and does not coincide with $h$, so that the restriction of $h-\widehat{R}_{h}^{I\left(\varphi_{1}\right) \backslash \Omega}$ to $\Omega$ is a positive harmonic function of finite order in $\Omega$ vanishing q.e. on the boundary of $\Omega$. Thus the theorem follows in this case, too.

\section{ACKNOWLEDGMENT}

The author thanks Pietro Poggi-Corradini for valuable comments and showing a preprint of his paper [12]. He also acknowledges helpful suggestions by the referee.

\section{REFERENCES}

1. H. Aikawa, Norm estimate of Green operator, perturbation of Green function and integrability of superharmonic functions, Math. Ann. 312 (1998), no. 2, 289-318. MR 99m:35042

2. A. Ancona, Une propriété de la compactification de Martin d'un domaine euclidien, Ann. Inst. Fourier (Grenoble) 29 (1979), no. 4, ix, 71-90. MR 81f:31013

3. $ـ$ Sur la frontière de Martin des domaines de Denjoy, Ann. Acad. Sci. Fenn. Ser. A I Math. 15 (1990), no. 2, 259-271. MR 92e:31001

4. D. H. Armitage and S. J. Gardiner, Classical potential theory, Springer-Verlag London Ltd., London, 2001. MR 2001m:31001

5. M. Benedicks, Positive harmonic functions vanishing on the boundary of certain domains in $\mathbf{R}^{n}$, Ark. Mat. 18 (1980), no. 1, 53-72. MR 82h:31004

6. N. Chevallier, Frontière de Martin d'un domaine de $\mathbf{R}^{n}$ dont le bord est inclus dans une hypersurface lipschitzienne, Ark. Mat. 27 (1989), no. 1, 29-48. MR 91e:31024

7. M. C. Cranston and T. S. Salisbury, Martin boundaries of sectorial domains, Ark. Mat. 31 (1993), no. 1, 27-49. MR 94k:31002

8. S. Friedland and W. K. Hayman, Eigenvalue inequalities for the Dirichlet problem on spheres and the growth of subharmonic functions, Comment. Math. Helv. 51 (1976), no. 2, 133-161. MR 54:568

9. S. J. Gardiner, Minimal harmonic functions on Denjoy domains, Proc. Amer. Math. Soc. 107 (1989), no. 4, 963-970. MR 90c:31013 
10. W. K. Hayman and P. B. Kennedy, Subharmonic functions. Vol. I, Academic Press, London, 1976. MR 57:665

11. A. Lömker, Martin boundaries of quasi-sectorial domains, Potential Anal. 13 (2000), no. 1, 11-67. MR 2002k:31023

12. P. Poggi-Corradini, On the failure of a generalized Denjoy-Wolff theorem, Conform. Geom. Dyn. 6 (2002), 13-32 (electronic). MR 2003a:30031

13. S. Segawa, Martin boundaries of Denjoy domains, Proc. Amer. Math. Soc. 103 (1988), no. 1, 177-183. MR 89m:31008

14. Martin boundaries of Denjoy domains and quasiconformal mappings, J. Math. Kyoto Univ. 30 (1990), no. 2, 297-316. MR 91j:30034

15. E. Sperner, Jr., Zur Symmetrisierung von Funktionen auf Sphären, Math. Z. 134 (1973), 317-327. MR 49:5310

Department of Mathematics, Shimane University, Matsue 690-8504, Japan

E-mail address: haikawa@math.shimane-u.ac.jp 